\title{
Hypomethylation of the CTCFL/BORIS promoter and aberrant expression during endometrial cancer progression suggests a role as an Epi-driver gene
}

\author{
Erling A. Hoivik ${ }^{1,2}$, Kanthida Kusonmano ${ }^{1,3}$, Mari K. Halle ${ }^{1,2}$, Anna Berg ${ }^{1,2}$, Elisabeth \\ Wik $^{4,5}$, Henrica M. J. Werner ${ }^{1}$, Kjell Petersen ${ }^{3}$, Anne M. Oyan ${ }^{2,6}$, Karl-Henning \\ Kalland $^{2,6}$, Camilla Krakstad ${ }^{1,2}$, Jone Trovik ${ }^{1,2}$, Martin Widschwendter7, Helga B. \\ Salvesen ${ }^{1,2}$ \\ ${ }^{1}$ Department of Obstetrics and Gynecology, Haukeland University Hospital, Bergen, Norway. \\ ${ }^{2}$ Centre for Cancer Biomarkers, Department of Clinical Science, University of Bergen, Bergen, Norway. \\ ${ }^{3}$ Computational Biology Unit, University of Bergen, Norway. \\ ${ }^{4}$ Centre for Cancer Biomarkers, Department of Clinical Medicine, University of Bergen, Bergen, Norway. Department of \\ Clinical Science, University of Bergen, Bergen, Norway. \\ ${ }^{5}$ Department of Pathology, Haukeland University Hospital, Bergen, Norway. \\ ${ }^{6}$ Department of Microbiology, Haukeland University Hospital, Bergen, Norway. \\ 7 Department of Women's Cancer, University College London Elizabeth Garrett Anderson Institute for Women's Health, \\ University College London, United Kingdom.
}

Correspondence to: Erling A. Hoivik, email: erling.hoivik@uib.no

Keywords: CTCFL/BORIS, CTCF, metastasis, recurrence, epi-driver gene

Received: December 13,2013 Accepted: January 26, $2014 \quad$ Published: January 28, 2014

This is an open-access article distributed under the terms of the Creative Commons Attribution License, which permits unrestricted use, distribution, and reproduction in any medium, provided the original author and source are credited.

ABSTRACT:

Cancers arise through accumulating genetic and epigenetic alterations, considered relevant for phenotype and approaches to targeting new therapies. We investigated a unique collection of endometrial cancer precursor samples and clinically annotated primary and metastatic lesions for two evolutionary and functionally related transcription factors, CCCTC-binding factor (zinc finger protein) (CTCF) and its paralogue CTCF-like factor, also denoted Brother of the Regulator of Imprinted Sites (CTCFL/BORIS). CTCF, a chromatin modeling- and transcription factor, is normally expressed in a ubiquitous fashion, while CTCFL/BORIS is restricted to the testis. In cancer, CTCF is thought to be a tumor suppressor, while CTCFL/BORIS has been suggested as an oncogene. CTCF mutations were identified in $13 \%$, with CTCF hotspot frameshift mutations at p.T204, all observed solely in the endometrioid subtype, but with no association with outcome. Interestingly, CTCFL/BORIS was amongst the top ranked genes differentially expressed between endometrioid and non-endometrioid tumors, and increasing mRNA level of CTCFL/BORIS was highly significantly associated with poor survival. As aberrant CTCFL/BORIS expression might relate to loss of methylation, we explored methylation status in clinical samples from complex atypical hyperplasia, through primary tumors to metastatic lesions, demonstrating a pattern of DNA methylation loss during disease development and progression in line with the increase in CTCFL/BORIS mRNA expression observed. Thus, CTCF and CTCFL/BORIS are found to diverge in the different subtypes of endometrial cancer, with CTCFL/BORIS activation through demethylation from precursors to metastatic lesions. We thus propose, CTCFL/BORIS as an Epi-driver gene in endometrial cancer, suggesting a potential for future vaccine development. 


\section{INTRODUCTION:}

Endometrial cancer (EC) of the uterine corpus, a common malignancy of the female genital tract, arises as a consequence of the accumulation of genetic and epigenetic alterations, under the influence of environmental and systemic factors [1-3]. For the majority of the patients, abnormal vaginal bleeding contributes to an early diagnosis, with the possibility to offer curative primary surgical treatment though restricted or extended surgical procedures [4]. Although overall survival rate is as high as $85 \%$ at 5 years [1], patients with metastatic endometrial cancer have poor prognosis, a median survival of approximately one year, and no improvement on survival over the last decades [4].

Endometrial cancer is traditionally classified into type I and type II subtypes. Type I cancers account for $80-85 \%$ of EC cases, are of endometrioid histology, more often well differentiated and associate with favorable prognosis. In contrast the type II cancers are non-endometrioid carcinomas, poorly differentiated and associate with poorer survival [1]. Although considerable overlap exists, the type I and II distinction is also supported by differences in molecular alterations: Microsatellite Instability (MSI), PTEN, KRAS and CTNNBI (encoding $\beta$-Catenin) mutations are often found in the type I, while TP53 mutations and ERBB2 (encoding HER-2) and MYC amplifications are more frequently detected in type II [1]. Also, loss of Estrogen (ERalpha) and Progesterone receptor (PR) is a feature of type II [4].

The CTCF CCCTC-binding factor (zinc finger protein) is a conserved transcription factor involved in gene regulation of a wide range of target genes, including the oncogene MYC and tumor suppressors such as BRCA1 and TP53 [5-8]. CTCF is also involved in chromosome "gymnastics", by looping chromatin to ensure that regulatory cis regions are positioned correctly for transcriptional activity [9]. Moreover, CTCF function as an insulator protein in $\mathrm{X}$-chromosome inactivation and imprinting, being important in developmental processes to define functional regions of the genome during mammalian development $[10,11]$. CTCF has a paralogue in the CTCF-like factor, also denoted Brother of the Regulator of Imprinted Sites (CTCFL/BORIS) [12]. The two factors display distinct mutual exclusive expression patterns; CTCF is ubiquitously expressed, except in testis, while CTCFL/BORIS expression is specific to germ cells of the testis $[12,13]$. The functional characteristics of CTCFL/ BORIS are less explored than those of its counterpart CTCF, but a role in setting the re-methylation marks on the genome of male germ cells during spermatogenesis, as well as in testicular growth and male fertility has been supported based on studies of experimental knockout mice $[12,14,15]$. CTCFL/BORIS fall into the category of Cancer/Testis (CT) antigens (CTCFL/BORIS; CT27), a group of tumor associated genes aberrantly expressed in many cancers, but with restricted expression confined to the testis in their normal state, thus belonging to a group of genes suggested to represent targets for future cancer vaccine development $[16,17]$.

In the present study we have performed comprehensive molecular profiling of a unique sample collection of fresh frozen endometrial cancer precursor lesions in parallel with clinically annotated primary and metastatic endometrial carcinoma lesions to explore potential links between disease progression and $C T C F$ mutations, aberrant expression of CTCFL/BORIS and CTCFL/BORIS DNA methylation. We report for the first time, that CTCF and CTCFL/BORIS seem to diverge in the different subtypes of endometrial cancer; with CTCF mutations occurring in endometrioid subtype and aberrant expression of CTCFL/BORIS being defined to cases of non-endometrioid subtype. Aberrant CTCFL/BORIS expression is induced through promoter hypomethylation in early steps of cancer progression, with increased mRNA expression from premalignant to primary tumors and further to metastatic lesions. This suggests CTCFL/BORIS as an Epi-driver gene with a potential for future vaccine development in endometrial cancer.

\section{RESULTS AND DISCUSSION:}

\section{CTCF p.T204 hotspot mutations found only in endometrioid tumors.}

Based on the key importance of CTCF in chromatin organization and transcriptional regulation, together with accumulating reports identifying CTCF mutations in cancer patients, we performed sequencing of all coding exons of $C T C F$ in 70 primary endometrial carcinomas, identifying a mutational frequency of $13 \%$ (Figure 1A). A recent cross-cancer study, has pointed to CTCF mutations being most frequently found in endometrial cancer (16.5 $\%$ ) across 12 major cancer types (overall frequency of 2.5 $\%$ [18]. The Sanger Catalogue of Somatic Mutations in Cancer (COSMIC) database, presents a mutation rate of $17 \%$ for endometrial cancer samples $(n=281)$ compared to $1.7 \%$ mutation rate across 43 cancer types explored (132 mutated in 7928 explored samples (Sanger database August 2013) [19]. Thus, we find a comparable and high frequency of CTCF mutations in endometrial carcinomas in line with the TCGA (The Cancer Genome Atlas) study and the Sanger database.

A recent study on patients with developmental disorders and intellectual disability, suggests a functional link between CTCF mutations and cognitive processes [20]; in contrast a functional descriptive link of CTCF mutations towards cancer is lacking. In our study, a cluster of four mutations was found in the C-terminal of the protein at AA positions 639-662, as well as a hotspot 
mutation site at p.T204 causing frameshift in four cases predicting severe impact on function (p.T204fs*18, p.T204fs*26; Fig 1A, B and Supplementary Table S1). Interestingly, a confirmation of the recurrent mutational site at p.T204 in endometrial cancer was published while our study was ongoing, reporting that this particular slippage mutation was frequent in MSI positive tumors [21]. In addition, both the Sanger database and the TCGA based pan cancer study suggest p.R377 as another hotspot mutational site, although we did not see this in our analysis [22].

It is interesting that our study and the TCGA data both reveal CTCF mutated cases present only in the endometrioid subtype. Although not reaching statistically significance in our data, the larger pan cancer study [18] confirms this as a statistically significant correlation, with no mutated cases in the serous/mixed classes (Supplementary Table S1 and Figure S1), although a single exception has been reported [23]. Despite this, CTCF mutations seem to be limited to cases with the lesser aggressive endometrioid histology.

Neither CTCF mutations, nor CTCF mRNA expression data, did significantly influence survival or associate with other clinico-pathological markers for outcome (Figure 1B, Supplementary Table S2-3). This was consistent with mutational and clinical data downloaded from TCGA [24].

\section{CTCFL/BORIS is differentially expressed in endometrioid compared to non-endometrioid tumors}

In light of the finding that all CTCF mutations were solely confined to the endometrioid subtype, we performed an analysis of significantly differently expressed genes
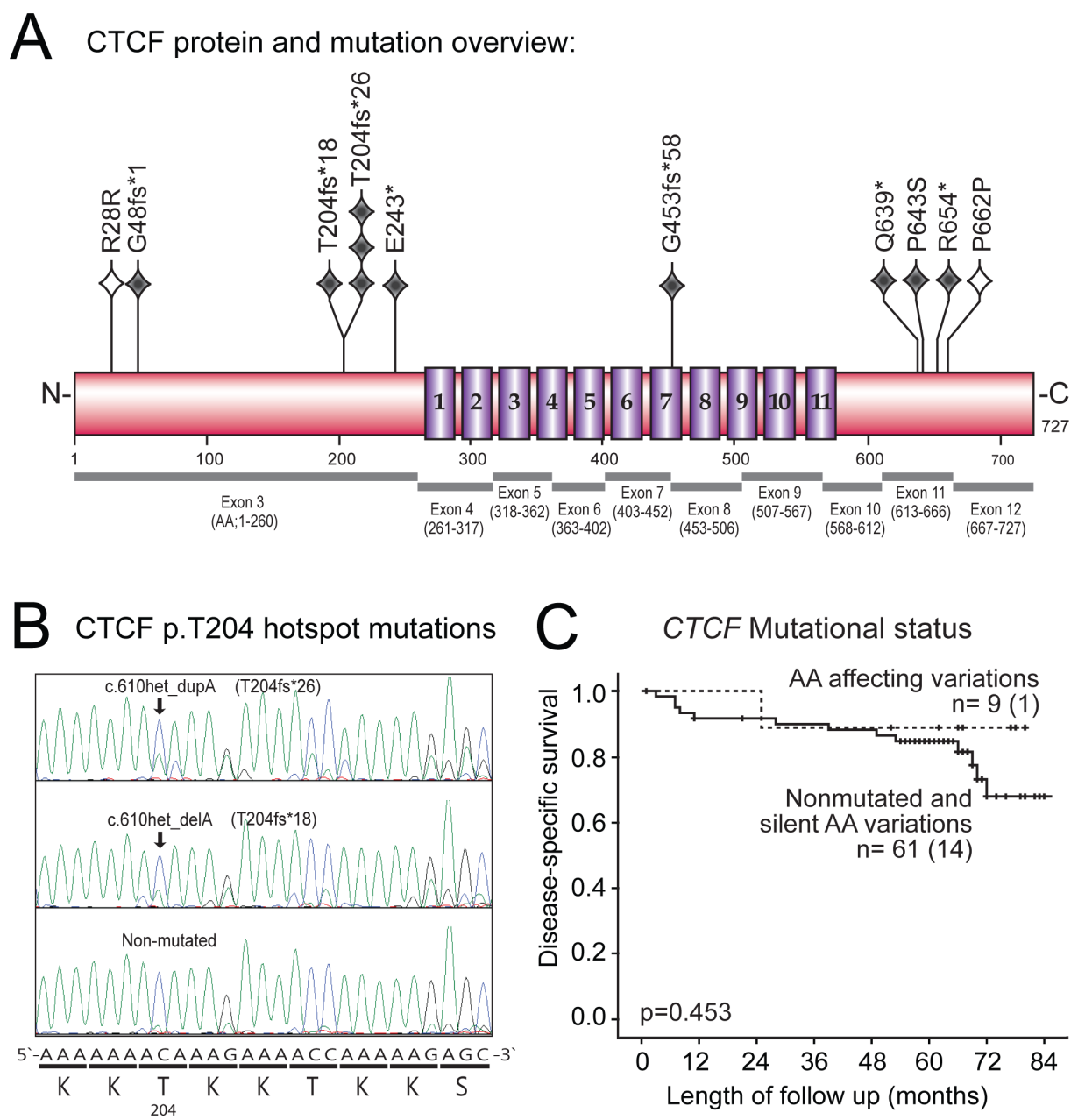

Figure 1: CTCF mutations, p.T204 hotspot site and association with disease-specific survival in primary endometrial carcinomas. (A) Schematic overview of the CTCF protein showing position of mutations relative to Zink-finger domains (numbered 1-11). Grey diamonds indicate nonsense/frameshift mutation, open diamonds indicate silent mutation, one diamond per mutation. Exons corresponding to translated protein regions are shown below protein structure. CTCF gene; NCBI RefSeq NM_006565, chromosome position chr16:67596310-67673088 on GRCh37/hg19 assembly. (B) Chromatogram of the hotspot mutations (arrows) at position c.610, causing frameshift mutations at corresponding protein position p.T204. Consensus DNA nucleotide sequence along with protein sequence is shown below. (C) Disease-specific survival plot for patients with or without $C T C F$ mutations indicates no significant differences in $C T C F$ mutated vs. non-mutated group. 
(SAM, Significance analysis of microarrays [25]) between endometrioid vs non-endometrioid subtypes. We discovered the CTCF-related factor named CTCFL/BORIS to be significantly increased in the non-endometrioid subtype (Among the top 417 genes [not shown] with estimated False Discovery Rate [FDR] $<0.001$, q-value $<0.001$, and fold change $\geq 2.000$, CTCFL/BORIS was ranked 24. ${ }^{\text {th }}$ with fold change of 2.257). Since the CTCFL/ BORIS is closely related to CTCF, has a potential role in methylation, and belong to a group of tumor associated genes (CT antigens) aberrantly expressed in many cancers, but with normal expression restricted to the testis, we were prompted to further investigate this factor in relation to tumor progression and clinical phenotypes.

\section{High CTCFL/BORIS level identifies aggressive endometrial carcinoma}

CTCFL/BORIS mRNA expression increases significantly through the stages of cancer development investigated, from complex atypical hyperplasia $(\mathrm{CAH})$ to primary tumors (PT) and metastatic lesions (M) (Figure $2 \mathrm{~A}$ and $\mathrm{C}$ ). All the clinically established markers for aggressive endometrial carcinoma including high age, high FIGO stage, non-endometrioid histology, high grade and hormone receptor loss were significantly associated with high CTCFL/BORIS mRNA levels as listed in Table 1. In line with this, the group of patients with high

A

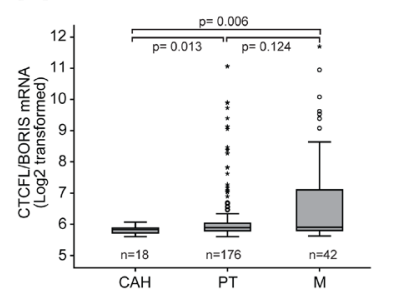

C
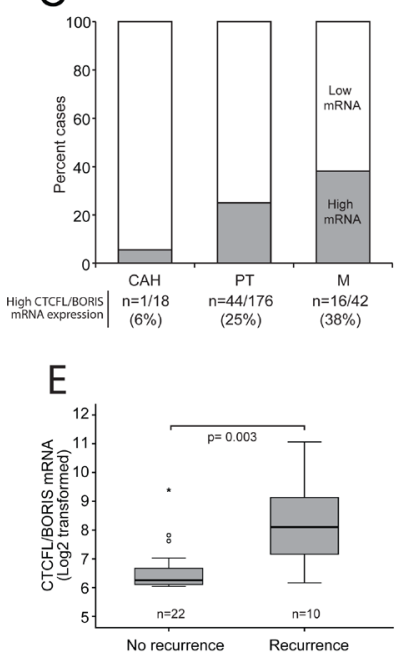

B

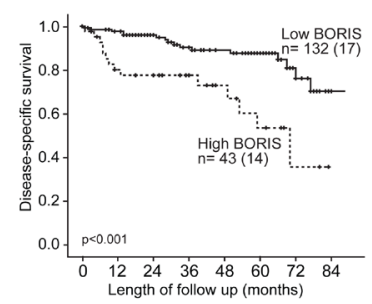

D

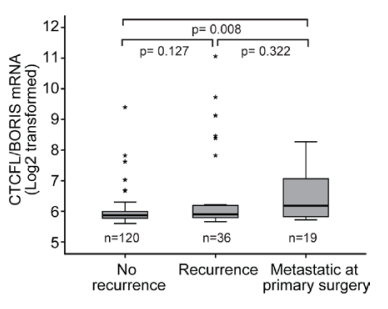

$\mathrm{F}$

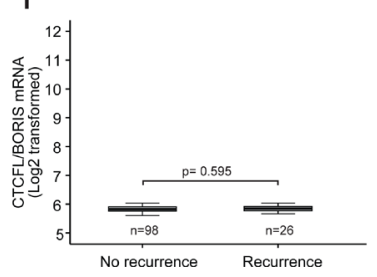

CTCFL/BORIS mRNA expression level, defined by the upper quartile, has a 5 year disease specific survival of $50 \%$ compared to $90 \%$ for the reminder (Figure 2B). To our knowledge this describes for the first time the aberrant expression of CTCFL/BORIS across these cancer developmental stages, and suggests the potential of CTCFL/BORIS as both a biomarker as well as a highly relevant target in metastatic endometrial cancer disease in particular.

Interestingly, CTCFL/BORIS, normally not expressed in the endometrium, was found to increase during cancer development and progression, which was also reflected in poor survival for patients with high expression levels. Samples from patients with no recurrence tended to have lower CTCFL/BORIS level compared to those who did recur (p-value of 0.127; Figure 2D), while primary tumors with metastatic disease at the time of primary surgery displayed a significantly higher level of CTCFL/BORIS (p-value of 0.008). Stratifying patients according to low vs. high CTCFL/BORIS levels, a significant higher expression level of CTCFL/BORIS was seen for recurrent cases only for the high expression group (p-value 0.003; Figure 2E and 2F).

\section{DNA methylation index (MI) of CTCFL/BORIS promoter decreases from premalignant to malignant lesions.}

We then hypothesized that the aberrant CTCFL/ BORIS expression might relate to loss of methylation as irregular CTCFL/BORIS expression is found in a range of tumorigenic cell lines and in several cancers including liver, breast, ovarian, prostate, colon, lung, endometrial cancers as well as melanoma and glioblastoma [26-28].

Figure 2: Increased CTCFL/BORIS mRNA expression level associates with cancer progression, poor survival. (A) CTCFL/BORIS mRNA expression increases significantly through the stages of cancer development and progression from complex atypical hyperplasia (CAH) to primary tumors (PT) to metastases (M). (B) High CTCFL/BORIS mRNA expression level (above upper quartile) identifies endometrial cancer patients with poor disease-specific survival. (C) Bar graph presentation showing proportions of cases with high CTCFL/ BORIS expression (above upper quartile) for the stages of cancer development and progression from CAH to PT to M. (D) Box plot presentation of CTCFL/BORIS mRNA levels in relation to systemic disease, i.e. development of recurrence or presence of non-resectable metastatic disease at primary surgery. (E, F) Box plot presentation of CTCFL/BORIS mRNA expression in relation to development of recurrent disease stratified for expression levels in primary tumors; compare high expression (E) to low (F) expression. A significant association between CTCFL/BORIS mRNA level and recurrent disease is seen only for the patient group with initial high CTCFL/BORIS mRNA level in primary lesions (E). 
Table 1: Clinico-pathological data in relation to CTCFL/BORIS mRNA expression in endometrial carcinomas.

\begin{tabular}{|c|c|c|c|c|}
\hline Variable $^{\mathrm{b}}$ & Category & CTCFL/BORIS Low n (\%) & CTCFL/BORIS High n (\%) & p-value \\
\hline \multirow{2}{*}{ Age } & $\leq 66$ & $71(85)$ & $13(15)$ & \multirow{2}{*}{0.009} \\
\hline & $>66$ & $61(66)$ & $31(34)$ & \\
\hline \multirow{4}{*}{ FIGO stage } & I & $100(80)$ & $26(21)$ & \multirow{4}{*}{0.001} \\
\hline & II & $12(92)$ & $1(8)$ & \\
\hline & III & $16(67)$ & $8(33)$ & \\
\hline & IV & $4(31)$ & $9(69)$ & \\
\hline \multirow{2}{*}{ Histological subtype } & Endometrioid & $119(84)$ & $23(16)$ & \multirow{2}{*}{$<0.001$} \\
\hline & Non-endometrioid & $13(38)$ & $21(62)$ & \\
\hline \multirow{2}{*}{ Histological grade } & Grade 1-2 & $86(85)$ & $15(15)$ & \multirow{2}{*}{$<0.001$} \\
\hline & Grade 3 & $45(62)$ & $28(38)$ & \\
\hline \multirow{2}{*}{$\mathrm{ER} \alpha(\mathrm{IHC})$} & High & $102(81)$ & $24(19)$ & \multirow{2}{*}{0.020} \\
\hline & Low & $26(62)$ & $16(38)$ & \\
\hline \multirow{2}{*}{ PR (IHC) } & High & $104(83)$ & $22(18)$ & \multirow{2}{*}{0.001} \\
\hline & Low & $25(56)$ & $20(44)$ & \\
\hline
\end{tabular}



${ }^{b}$ Data missing (n patients); Histological grade (2), ER $\alpha$ (8), PR (5).

One possible mechanism of the increased CTCFL/BORIS expression might be due to loss of epigenetic traits during cancer development/progression, a well-established element in inducing aberrant $\mathrm{CT}$ antigen expression in somatic tissues $[17,29,30]$. We therefore characterized DNA methylation status of the CTCFL/BORIS promoter,

A BSP target region:
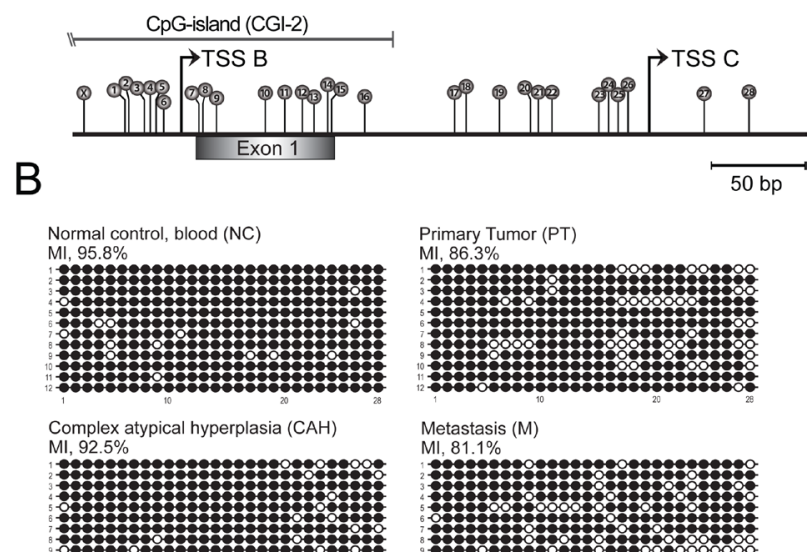

Unmethylated control, PCR amplified case MI, $1.8 \%$

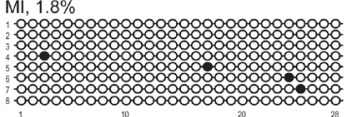

Primary Tumor (PT)

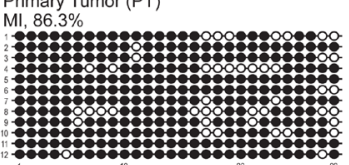

Metastasis (M)

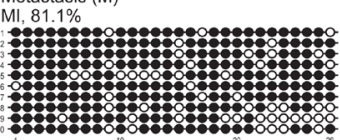

Unmethylated CpG-site

- Methylated CpG-site

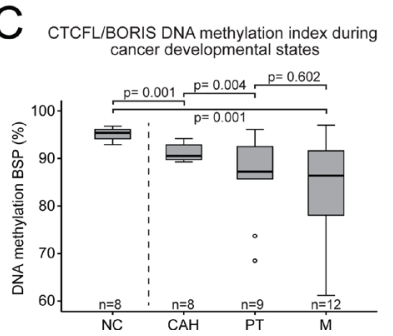

spanning across transcriptional start sites of alternative B- and C- promoters [14, 28] by bisulphite sequencing analysis on tissues representing various stages of cancer development (Figures 3A and S2A). Complex atypical hyperplasias, primary tumors and metastatic samples were compared to normal control (NC, buffy coat fraction from blood samples). As the fine mapping of the methylation status of the single $\mathrm{CpG}$-sites within the target did not

Figure 3: DNA methylation index (MI) shows decreasing level of CTCFL/BORIS promoter methylation from premalignant through primary to metastatic endometrial carcinoma lesions. (A) Overview of CpG-sites marked as lollipops numbered 1-28, on CTCFL/ BORIS promoter targeted in the bisulphite sequencing assay. Site marked X not included due to integration in PCR primer. The target region contains two dominant transcription start sites: TSS B and TSS C and exon 1 of the gene (extended overview in Figure S2). (B) Representative bisulphite sequencing samples of normal control (NC), complex atypical hyperplasias (CAH), primary tumors (PT) and metastasis (M). The CTCFL/BORIS promoter shows a gradual loss of methylation through cancer developmental stages compared to normal control (from blood buffy coat). Each horizontal line represents an epiallele, with each individual $\mathrm{CpG}$-site shown as either methylated $\mathrm{CpG}$ sites (black circles) or unmethylated $\mathrm{CpG}$ site (open circles), with positions corresponding to numbering in A. The vertical stack of epialleles describes analysis of multiple colonies/clones. The controls showed a high degree of methylation of mean value of $95.1 \%$, while an in vitro PCR generated unmethylated control was $1.8 \%$ methylated, indicating robust analysis. Analysis close to mean values in C) was selected for display. (C) Comprehensive box-plot of MI-values significantly declining through cancer progression with mean values for normal controls of $95.1 \%$, hyperplasias with atypia $91.2 \%$, primary tumors $86.2 \%$ and metastases $83.8 \%$. 
Table 2: Selected genes differentially expressed in groups based on low and high CTCFL/BORIS expression

\begin{tabular}{|l|l|l|l|}
\hline \multirow{2}{*}{ Regulation } & Gene Name & Description & Fold Change \\
\hline \multirow{3}{*}{ UP } & IGF2BP1 & Insulin-like growth factor 2 mRNA-binding protein 1 & 2.529 \\
\cline { 2 - 4 } & IGF2BP2 & Insulin-like growth factor 2 mRNA-binding protein 2 & 2.620 \\
\cline { 2 - 4 } & SOX11 & SRY (sex determining region Y)-box 11 & 2.343 \\
\hline \multirow{4}{*}{ DOWN } & ESR1 & Estrogen receptor $\alpha$ & 3.501 \\
\cline { 2 - 4 } & PGR & Progesterone receptor & 2.993 \\
\cline { 2 - 4 } & AR & Androgen receptor & 2.067 \\
\cline { 2 - 4 } & SOX17 & SRY (sex determining region Y)-box 17 & 2.180 \\
\hline
\end{tabular}

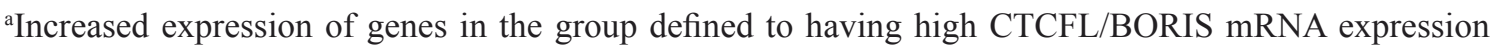
(according to upper quartile limits), compared to the group of low CTCFL/BORIS mRNA expression. ${ }^{b}$ Reduced expression of genes in the group defined to having high CTCFL/BORIS mRNA expression, compared to the group of cases classified with low CTCFL/BORIS mRNA expression.

reveal a distinct demethylation pattern, (Figure 3B), a methylation index (MI) was calculated for each specimen and demonstrated a significant reduction in the DNA methylation level during the stages of cancer development, most pronounced for the step from complex atypical hyperplasias to primary tumors. Although not reaching statistical significance, the metastatic sample set showed a tendency to less CTCFL/BORIS promoter methylation compared to primary tumors exploring samples investigated by k27 array (Illumina Infinium Human Methylation27 BeadChip array) (Supplementary Figure S2C) also inversely mirroring RNA expression levels (Figure 2A). There was a significant correlation between the results from the MI and available data for k27 assay for overlapping samples (Supplementary Figure S2C). Taken together, the methylation pattern observed appears to be in line with the general notion that hypomethylation occurs as an early step in cancer [30].

\section{High CTCFL/BORIS level and loss of CTCFL/ BORIS promoter methylation associate with aggressive subgroups in external data}

We further explored expression levels and methylation pattern for CTCFL/BORIS in TCGA data for primary tumors for the methylation assay k27 and Agilent $244 \mathrm{k}$ custom gene expression microarray. We validated a similar significant correlation between high CTCFL/BORIS mRNA expression and non-endometrioid and grade 3 subgroups in the TCGA data (Supplementary Figure S3A). Interestingly, in the TCGA data we also find a significant correlation between CTCFL/BORIS hypomethylation and non-endometrioid histology and grade 3 inversely mirroring RNA expression levels (Supplementary Figure S3B).

\section{Hormone receptor loss associates with high CTCFL/BORIS expression}

Selected genes found to be highly differentially expressed comparing CTCFL/BORIS low vs. high mRNA microarray expression levels in primary tumors, is presented in Table 2 and as a full list in Supplementary Table S4. Interestingly, genes encoding estrogen- (ESR1), progesterone- (PGR) and androgen (AR) receptors were all down-regulated in the group expressing CTCFL/BORIS at high mRNA levels (defined by upper quartile limit). This fits well with the long time reported association between loss of ERalpha/PR expression and aggressive disease [31, 32]. Androgen receptor has been found to have anti-proliferative effects in the normal endometrium, and therefore loss of functional AR might promote carcinogenesis [33].

\section{CTCFL/BORIS - a potential Epi-driver gene in endometrial cancer?}

A study investigating the genome-wide CTCF and CTCFL/BORIS binding to chromatin in ChIP(Chromatin ImmunoPrecipitation)-experiments in cell lines, reveals that CTCFL/BORIS almost exclusively binds to CTCF consensus sites at promoters suggesting an important regulatory function [15]. Extrapolating this to cancer proposes that aberrant expression of CTCFL/BORIS might overrule genes normally regulated by $\mathrm{CTCF}$, or at least compete with CTCF on transcriptional target genes with different outcome in regulation due to differences in $\mathrm{N}$ and $\mathrm{C}$-terminals and discrete protein interaction partners [12-15, 34, 35]. Exogenous expression of CTCFL/BORIS in cell systems induced the expression of hTERT, a subunit of the telomerase enzyme conferring immortality in tumorigenesis, suggesting one mechanism through which 
CTCFL/BORIS may lead to increase in proliferation in cancer [36].

The concept of Epi-driver genes is fairly new, and we propose for the first time CTCFL/BORIS as an potential Epi-driver gene according to the criteria suggested by Vogelstein [37] based on the following; Epigenetic alterations characterized by promoter hypomethylation lead to aberrant expression of CTCFL/BORIS mRNA expression level in cancer, cancerous expression is clearly associated with clinical phenotypes and cancer progression, it is not frequently mutated and its related factor CTCF (analogous to CTCFL/BORIS) has recently been suggested to be a Mut-driver gene in endometrial cancer $[18,38]$.

There are several aspects of Epi-driver genes that are potentially beneficial in therapeutic approaches. First, as epigenetic mechanisms are inducing aberrant expression of Epi-driver genes, it also suggests that future specific epigenetic modulator drugs might be able to target specific epigenetic changes causing this, as epigenetic changes are potentially reversible [39, 40]. Second, it also suggests that Epi-drivers might be targeted by cancer vaccines, as has been suggested for Cancer/Testis antigens [17]. In fact, through a metastatic mammary mouse model, a BORIS-based cancer vaccine delivered by dendritic cells was shown to be effective in inhibiting tumor growth and the formation of metastasis [41]. Clinical trials targeting CT genes are few, but NY-ESO-1 (New York esophageal squamous cell carcinoma 1, also known as CTAGB1, CT6.1) was targeted by adoptive immunotherapy employing genetically modified lymphocytes in patients with metastatic synovial cell sarcoma or melanoma patients and was shown to be effective with impressive responses to treatment with no apparent toxicity effect (Effective responses of $67 \%$ and $45 \%$ ) [42]. Indeed, the idea of targeting the testis-specific genes normally not expressed in females, but in systemic endometrial carcinomas, through cancer vaccines, is appealing.

\section{MATERIAL AND METHODS:}

\section{Patient material ethical approvals}

Patients were included in the study after written informed consent, approved by the Norwegian Data Inspectorate, Norwegian Social sciences Data Services, the Regional Research Ethics Committee in Medicine and the local Hospital (NSD15501; REK 052.01). Samples were collected from patients treated at the Haukeland University Hospital, Norway, as previously described [31]. Tumors were snap frozen in liquid nitrogen (1) and stored at minus $80^{\circ} \mathrm{C}$ until validation of $>50 \%$ purity for the malignant epithelial component before RNA or DNA extraction. Blood (buffy coat) was collected from normal healthy individuals, age matched to the EC group of patients, for DNA extraction to be used as control samples for the DNA methylation analyses.

\section{RNA extraction and RNA analysis}

RNA was extracted with the RNAeasy tissue kit from Qiagen, following the standard protocol as recommended. Cy3-cRNA labeled samples were hybridized to Agilent Whole Human Genome Microarrays 44k array (Cat. nr.G4112F) according to the manufacturers' instructions, and scanned employing the Agilent Microarray Scanner Bundle as previously described [31]. The arrays were quantile normalized using median spot signal, log2 transformed. Statistical analysis of differentially expressed genes (SAM) was performed with the J-Express software [25]. The cutoff levels for low- and high CTCFL/BORIS mRNA expression was determined based on quartile limits merging groups with similar survival in Kaplan-Meier survival analysis. In this, as well as support from the CTCFL/BORIS literature $[14,43]$, we assume that cases classified within the group of low CTCFL/BORIS mRNA expression, do not express CTCFL/BORIS or alternatively at low levels, although we did not perform additional RTPCR analysis to confirm this.

\section{DNA extraction, sequencing and methylation analysis}

Genomic DNA (gDNA) was extracted after proteinase $\mathrm{K}$ digestion, and precipitated following a standard protocol. gDNA was bisulphite treated, amplified and hybridized employing the Illumina Infinium HumanMethylation27 BeadChip kit (see [44] for details). For sequencing, 10 ng gDNA was whole genome amplified by employing the GenomePlex Complete Whole Genome Amplification (WGA) kit (Sigma) with the GenElute PCR Clean-Up kit (Sigma) for purification. Amplicons for direct sequencing were obtained by PCR with $25 \mathrm{ng}$ WGA-material with HotSTAR polymerase (Qiagen) and primers according to Supplementary Table S5. After agarose-gel purification on Qiagen gel-extraction kit, amplicons (all coding exons of CTCF) were sequenced by universal M13-primers utilizing the BigDye Terminator Sequencing Kit, version 1.3, analyzed on an ABI Prism 3100 genetic analyzer (Applied Biosystems). Sequences were inspected with Sequence Scanner software 1.0 (Applied Biosystems), excluding variants present in the SNP database (dbSNP) http://www.ncbi.nlm.nih.gov/ snp). Mutational data were compared against the Sanger COSMIC database (http://www.cancer.sanger.ac.uk/ cancergenome/projects/cosmic/), as well as TCGA data (http://www.cancergenome.nih.gov). Methylation analysis of CTCFL/BORIS NCBI RefSeq NR_072975, spanning nucleotides chr20:56099842-56100217 on GRCh37/ 
hg19 assembly was performed in bisulphite sequencing assay. $200 \mathrm{ng}$ of gDNA was treated in DNA methylation Direct kit by Zymo Research, following PCR by specific primers (available upon request) employing HotSTAR polymerase (Qiagen), and subcloning in pGEM-T-easy vector (Promega). Up to 12 colonies were sequenced from each tumor/case for the analysis of $\mathrm{CpG}$-site specific DNA methylation in the CTCFL/BORIS promoter regions. Methylation positive and methylation depleted control PCR products (primers available upon request) spanning the CTCFL/BORIS promoter region were gel purified and in vitro methylated in the presence or absence of the SssI CpG methylase (New England Biolabs, United Kingdom) at $37^{\circ} \mathrm{C}$ for four hours with SssI (2 U/ $\mu$ g DNA) in presence of S-adenosylmethionine (SAM) $(160 \mu \mathrm{M})$, with additional SssI $(0.3 \mathrm{U} / \mu \mathrm{g}$ DNA) and SAM $(160 \mu \mathrm{M})$ after two hours. Methylation indexes (MI) were calculated as a percentage of the methylated CpGs compared to the total $\mathrm{CpGs}$ in each analysis. For matters related to the Illumina Infinium Human Methylation27 BeadChip array, it was performed as described in reference [44].

\section{Statistical analysis}

The Fischer exact tests was used to test the significance of associations between mutations within subtypes, employing the SPSS 18.0 statistical software. Alternatively, when appropriate, Pearson Chi-square exact two-sided tests were employed. All statistical tests were considered significant when $\mathrm{p}$-values $\leq 0.05$. Univariate survival analyses of time to death due to endometrial carcinoma (disease specific survival) were performed using the Kaplan-Meier method. Entry date was the date of primary surgery. Patients who died from other causes were censored at the date of death. Differences in survival between groups were estimated by two-sided log-rank (Mantel Cox) tests.

\section{ACKNOWLEDGEMENTS:}

We are grateful for excellent technical assistance of Britt Edvardsen, Hua My Hoang, Kadri Madissoo and Ellen Valen, and to the patients for their willingness to participate in scientific studies.

\section{REFERENCES:}

1. Di Cristofano A and Ellenson LH. Endometrial carcinoma. Annual review of pathology. 2007; 2:57-85.

2. Tao MH and Freudenheim JL. DNA methylation in endometrial cancer. Epigenetics : official journal of the DNA Methylation Society. 2010; 5(6):491-498.

3. Jones A, Teschendorff AE, Li Q, Hayward JD, Kannan A, Mould T, West J, Zikan M, Cibula D, Fiegl H, Lee SH, Wik E, Hadwin R, Arora R, Lemech C, Turunen H, et al. Role of
DNA Methylation and Epigenetic Silencing of HAND2 in Endometrial Cancer Development. PLoS medicine. 2013; 10(11):e1001551.

4. Salvesen HB, Haldorsen IS and Trovik J. Markers for individualised therapy in endometrial carcinoma. The lancet oncology. 2012; 13(8):e353-361.

5. Fiorentino FP and Giordano A. The tumor suppressor role of CTCF. Journal of cellular physiology. 2012; 227(2):479492.

6. Recillas-Targa F, de la Rosa-Velazquez IA and Soto-Reyes E. Insulation of tumor suppressor genes by the nuclear factor CTCF. Biochemistry and cell biology = Biochimie et biologie cellulaire. 2011; 89(5):479-488.

7. Lobanenkov VV, Nicolas RH, Adler VV, Paterson H, Klenova EM, Polotskaja AV and Goodwin GH. A novel sequence-specific DNA binding protein which interacts with three regularly spaced direct repeats of the CCCTCmotif in the 5'-flanking sequence of the chicken c-myc gene. Oncogene. 1990; 5(12):1743-1753.

8. Filippova GN, Fagerlie S, Klenova EM, Myers C, Dehner Y, Goodwin G, Neiman PE, Collins SJ and Lobanenkov VV. An exceptionally conserved transcriptional repressor, CTCF, employs different combinations of zinc fingers to bind diverged promoter sequences of avian and mammalian c-myc oncogenes. Molecular and cellular biology. 1996; 16(6):2802-2813.

9. Merkenschlager M and Odom DT. CTCF and cohesin: linking gene regulatory elements with their targets. Cell. 2013; 152(6):1285-1297.

10. Herold M, Bartkuhn M and Renkawitz R. CTCF: insights into insulator function during development. Development. 2012; 139(6):1045-1057.

11. Yang $\mathrm{J}$ and Corces VG. Chromatin insulators: a role in nuclear organization and gene expression. Advances in cancer research. 2011; 110:43-76.

12. Klenova EM, Morse HC, 3rd, Ohlsson R and Lobanenkov VV. The novel BORIS + CTCF gene family is uniquely involved in the epigenetics of normal biology and cancer. Seminars in cancer biology. 2002; 12(5):399-414.

13. Ohlsson R, Lobanenkov V and Klenova E. Does CTCF mediate between nuclear organization and gene expression? BioEssays : news and reviews in molecular, cellular and developmental biology. 2010; 32(1):37-50.

14. Pugacheva EM, Suzuki T, Pack SD, Kosaka-Suzuki N, Yoon J, Vostrov AA, Barsov E, Strunnikov AV, Morse HC, 3rd, Loukinov D and Lobanenkov V. The structural complexity of the human BORIS gene in gametogenesis and cancer. PloS one. 2010; 5(11):e13872.

15. Sleutels F, Soochit W, Bartkuhn M, Heath H, Dienstbach S, Bergmaier P, Franke V, Rosa-Garrido M, van de Nobelen S, Caesar L, van der Reijden M, Bryne JC, van Ijcken W, Grootegoed JA, Delgado MD, Lenhard B, et al. The male germ cell gene regulator CTCFL is functionally different from CTCF and binds CTCF-like consensus sites in a 
nucleosome composition-dependent manner. Epigenetics \& chromatin. 2012; 5(1):8.

16. Kulkarni P, Shiraishi T, Rajagopalan K, Kim R, Mooney $\mathrm{SM}$ and Getzenberg RH. Cancer/testis antigens and urological malignancies. Nature reviews Urology. 2012; 9(7):386-396.

17. Simpson AJ, Caballero OL, Jungbluth A, Chen YT and Old LJ. Cancer/testis antigens, gametogenesis and cancer. Nature reviews Cancer. 2005; 5(8):615-625.

18. Cancer Genome Atlas Research N, Genome Characterization $\mathrm{C}$, Chang $\mathrm{K}$, Creighton CJ, Davis C, Donehower L, Drummond J, Wheeler D, Ally A, Balasundaram M, Birol I, Butterfield YS, Chu A, Chuah E, Chun HJ, Dhalla N, et al. The Cancer Genome Atlas Pan-Cancer analysis project. Nature genetics. 2013; 45(10):1113-1120.

19. Forbes SA, Bindal N, Bamford S, Cole C, Kok CY, Beare D, Jia M, Shepherd R, Leung K, Menzies A, Teague JW, Campbell PJ, Stratton MR and Futreal PA. COSMIC: mining complete cancer genomes in the Catalogue of Somatic Mutations in Cancer. Nucleic acids research. 2011; 39(Database issue):D945-950.

20. Gregor A, Oti M, Kouwenhoven EN, Hoyer J, Sticht H, Ekici AB, Kjaergaard S, Rauch A, Stunnenberg HG, Uebe $\mathrm{S}$, Vasileiou G, Reis A, Zhou H and Zweier C. De novo mutations in the genome organizer CTCF cause intellectual disability. American journal of human genetics. 2013; 93(1):124-131.

21. Zighelboim I, Mutch DG, Knapp A, Ding L, Xie M, Cohn DE and Goodfellow PJ. High Frequency Strand Slippage Mutations in CTCF in MSI-Positive Endometrial Cancers. Human mutation. 2013.

22. Ciriello G, Miller ML, Aksoy BA, Senbabaoglu Y, Schultz $\mathrm{N}$ and Sander C. Emerging landscape of oncogenic signatures across human cancers. Nature genetics. 2013; 45(10):1127-1133.

23. Le Gallo M, O'Hara AJ, Rudd ML, Urick ME, Hansen NF, O’Neil NJ, Price JC, Zhang S, England BM, Godwin AK, Sgroi DC, Program NIHISCCS, Hieter P, Mullikin $\mathrm{JC}$, Merino MJ and Bell DW. Exome sequencing of serous endometrial tumors identifies recurrent somatic mutations in chromatin-remodeling and ubiquitin ligase complex genes. Nature genetics. 2012; 44(12):1310-1315.

24. Goldman M, Craft B, Swatloski T, Ellrott K, Cline M, Diekhans M, Ma S, Wilks C, Stuart J, Haussler D and Zhu J. The UCSC Cancer Genomics Browser: update 2013. Nucleic acids research. 2013; 41(Database issue):D949-954.

25. Stavrum AK, Petersen K, Jonassen I and Dysvik B. Analysis of gene-expression data using J-Express. Current protocols in bioinformatics / editoral board, Andreas D Baxevanis [et al]. 2008; Chapter 7:Unit 73.

26. Chen K, Huang W, Huang B, Wei Y, Li B, Ge Y and Qin Y. BORIS, brother of the regulator of imprinted sites, is aberrantly expressed in hepatocellular carcinoma. Genetic testing and molecular biomarkers. 2013; 17(2):160-165.

27. Martin-Kleiner I. BORIS in human cancers -- a review. European journal of cancer. 2012; 48(6):929-935.

28. Renaud S, Pugacheva EM, Delgado MD, Braunschweig R, Abdullaev Z, Loukinov D, Benhattar J and Lobanenkov $\mathrm{V}$. Expression of the CTCF-paralogous cancer-testis gene, brother of the regulator of imprinted sites (BORIS), is regulated by three alternative promoters modulated by $\mathrm{CpG}$ methylation and by CTCF and p53 transcription factors. Nucleic acids research. 2007; 35(21):7372-7388.

29. Garraway LA and Lander ES. Lessons from the cancer genome. Cell. 2013; 153(1):17-37.

30. Rodriguez-Paredes M and Esteller M. Cancer epigenetics reaches mainstream oncology. Nature medicine. 2011; 17(3):330-339.

31. Wik E, Raeder MB, Krakstad C, Trovik J, Birkeland E, Hoivik EA, Mjos S, Werner HM, Mannelqvist M, Stefansson IM, Oyan AM, Kalland KH, Akslen LA and Salvesen HB. Lack of estrogen receptor-alpha is associated with epithelial-mesenchymal transition and PI3K alterations in endometrial carcinoma. Clinical cancer research : an official journal of the American Association for Cancer Research. 2013; 19(5):1094-1105.

32. Trovik J, Wik E, Werner HM, Krakstad C, Helland H, Vandenput I, Njolstad TS, Stefansson IM, Marcickiewicz J, Tingulstad S, Staff AC, MoMa TECsg, Amant F, Akslen LA and Salvesen HB. Hormone receptor loss in endometrial carcinoma curettage predicts lymph node metastasis and poor outcome in prospective multicentre trial. European journal of cancer. 2013; 49(16):3431-3441.

33. Cloke $\mathrm{B}$ and Christian M. The role of androgens and the androgen receptor in cycling endometrium. Molecular and cellular endocrinology. 2012; 358(2):166-175.

34. Vatolin S, Abdullaev Z, Pack SD, Flanagan PT, Custer M, Loukinov DI, Pugacheva E, Hong JA, Morse H, 3rd, Schrump DS, Risinger JI, Barrett JC and Lobanenkov VV. Conditional expression of the CTCF-paralogous transcriptional factor BORIS in normal cells results in demethylation and derepression of MAGE-A1 and reactivation of other cancer-testis genes. Cancer research. 2005; 65(17):7751-7762.

35. Hong JA, Kang Y, Abdullaev Z, Flanagan PT, Pack SD, Fischette MR, Adnani MT, Loukinov DI, Vatolin S, Risinger JI, Custer M, Chen GA, Zhao M, Nguyen DM, Barrett JC, Lobanenkov VV, et al. Reciprocal binding of CTCF and BORIS to the NY-ESO-1 promoter coincides with derepression of this cancer-testis gene in lung cancer cells. Cancer research. 2005; 65(17):7763-7774.

36. Renaud S, Loukinov D, Alberti L, Vostrov A, Kwon YW, Bosman FT, Lobanenkov V and Benhattar J. BORIS/ CTCFL-mediated transcriptional regulation of the hTERT telomerase gene in testicular and ovarian tumor cells. Nucleic acids research. 2011; 39(3):862-873.

37. Vogelstein B, Papadopoulos N, Velculescu VE, Zhou S, 
Diaz LA, Jr. and Kinzler KW. Cancer genome landscapes. Science. 2013; 339(6127):1546-1558.

38. Tamborero D, Gonzalez-Perez A, Perez-Llamas C, DeuPons J, Kandoth C, Reimand J, Lawrence MS, Getz G, Bader GD, Ding L and Lopez-Bigas N. Comprehensive identification of mutational cancer driver genes across 12 tumor types. Scientific reports. 2013; 3:2650.

39. Gronbaek K, Hother C and Jones PA. Epigenetic changes in cancer. APMIS : acta pathologica, microbiologica, et immunologica Scandinavica. 2007; 115(10):1039-1059.

40. Dawson MA and Kouzarides T. Cancer epigenetics: from mechanism to therapy. Cell. 2012; 150(1):12-27.

41. Mkrtichyan M, Ghochikyan A, Davtyan H, Movsesyan N, Loukinov D, Lobanenkov V, Cribbs DH, Laust AK, Nelson EL and Agadjanyan MG. Cancer-testis antigen, BORIS based vaccine delivered by dendritic cells is extremely effective against a very aggressive and highly metastatic mouse mammary carcinoma. Cellular immunology. 2011; 270(2):188-197.

42. Robbins PF, Morgan RA, Feldman SA, Yang JC, Sherry RM, Dudley ME, Wunderlich JR, Nahvi AV, Helman LJ, Mackall CL, Kammula US, Hughes MS, Restifo NP, Raffeld M, Lee CC, Levy CL, et al. Tumor regression in patients with metastatic synovial cell sarcoma and melanoma using genetically engineered lymphocytes reactive with NY-ESO-1. Journal of clinical oncology : official journal of the American Society of Clinical Oncology. 2011; 29(7):917-924.

43. Loukinov DI, Pugacheva E, Vatolin S, Pack SD, Moon H, Chernukhin I, Mannan P, Larsson E, Kanduri C, Vostrov AA, Cui H, Niemitz EL, Rasko JE, Docquier FM, Kistler $\mathrm{M}$, Breen JJ, et al. BORIS, a novel male germ-line-specific protein associated with epigenetic reprogramming events, shares the same 11-zinc-finger domain with CTCF, the insulator protein involved in reading imprinting marks in the soma. Proceedings of the National Academy of Sciences of the United States of America. 2002; 99(10):6806-6811.

44. Zhuang J, Jones A, Lee SH, Ng E, Fiegl H, Zikan M, Cibula D, Sargent A, Salvesen HB, Jacobs IJ, Kitchener HC, Teschendorff AE and Widschwendter M. The dynamics and prognostic potential of DNA methylation changes at stem cell gene loci in women's cancer. PLoS genetics. 2012; 8(2):e1002517. 